Die Familie im Sozialrecht 


\title{
Sozialleistungen und Familie
}

\author{
Eberhard Eichenhofer
}

I. Einleitung

II. Dogmatik 354

III. Der Wandel $\quad 356$

IV. Wandel des Familien- und familienfördernden Sozialrechts 357

V. Familien- und Sozialrecht im Zeichen der Gleichbehandlung der Geschlechter 359

VI. Familienförderung - Eheförderung 361

VII. Familienförderung - neues Aufgabenfeld des Sozialrechts 363

VIII. Sozialrechtliche Ermöglichung und Begleitung von Elternschaft 365

IX. Familienförderung und Chancengleichheit für Familien 368

$\begin{array}{ll}X . \text { Fazit } & 370\end{array}$

\section{Einleitung}

Hans F. Zacher prägte zur Beschreibung des Verhältnisses von Ehe, Familie und Sozialrecht das Bild von der doppelten „Formung des Lebens“ durch „Zwei Schalen des Rechts“ - „der Ebene des vorfindlichen Rechts und der Ebene des Sozialrechts“. ${ }^{1}$ Als vorfindlich sei demgemäß das Familienrecht und als zweite Schale das die Familienförderung bezweckende Sozialrecht zu bestimmen. Diese Einsicht von Gewicht gibt den weiteren Überlegungen den Rahmen vor. Denn die Familie lässt sich nicht als eine rechtlich umhegte Naturgegebenheit begreifen, ${ }^{2}$ sondern ist und war seit langem dem Schutz und der Förderung durch die Allgemeinheit überantwortet. Das vorfindliche Privatrecht der Familie war seit alters von rechtseigenen Maßstäben bestimmt und auch das

1 Zacher, Ehe und Familie in der Sozialrechtsordnung, in: ders., Abhandlungen zum Sozialrecht, Heidelberg 1995, S. 555, 561.

2 Dies entsprach noch der Vorstellung der Familie im Römischen Recht. „Ius naturale est, quod natura omnia animalia docuit, (...) hinc descendit maris atque feminae coniugatio, quam nos matrimonium appellamus, hinc liberorum procreatio et educatio“ - so Vorspruch II der Institutionen. 
es umgebende Sozialrecht war von dem Bemühen getragen, die Familie im Blick auf deren allgemeine Aufgabe zu fördern.

Wer sich dieser Thematik zu Beginn des 21. Jahrhunderts rechtsvergleichend und im Interesse rechtsdogmatischer Vergewisserung annimmt, wird sich des Eindrucks schwerlich erwehren können, dass in diesem Jahrzehnt kein anderes Teilgebiet des Sozialrechts einen ähnlich tiefgreifenden Wandel er- und durchlebt wie die Familienförderung. Sie steht demgemäß auch nicht eben zufällig im Mittelpunkt der aktuellen rechtspolitischen Bemühungen - hierzulande wie anderwärts. Der Beitrag bewertet diese Politik nicht, sondern versucht, deren rechtssystematische Ergebnisse festzustellen, zu verstehen und ihre Folgen für das Gesamtsystem des Sozialrechts zu bestimmen. Darin liegt die Aufgabe von Rechtswissenschaft. ${ }^{3}$

\section{Dogmatik}

Den „Sinn von Normen des positiven Rechts“4 verstehend zu erschließen, Wertungsin Erkenntnis- und Wahrheitsfragen im Sinne „,wertungsneutraler Begriffsarbeit“ zu überführen, ${ }^{5}$ die „Nichtnegierbarkeit der Ausgangspunkte von Argumentationsketten“6 als Eigenheit zu akzeptieren und sich an die ,wissenschaftliche Überprüfung von Rechtssetzungsbehauptungen innerhalb einer Rechtsordnung auf der Grundlage geltender Rechtssätze"7 zu begeben, dies alles ist jedem wissenschaftlichen Bemühen um das Recht gemeinsam. Gerade im Sozialrecht ist das Recht als das Ergebnis einer bewussten Setzung zu verstehen. Als das Ergebnis des Widerstreits gesellschaftlicher Interessen findet es im gesetzten Recht seinen Ausgangs- und Endpunkt. Der Rechtspositivismus ist deshalb die konzeptionelle Basis aller Sozialrechtswissenschaft. In deren Bemühen um Begriffs-, Theorie- und Systembildung ${ }^{8}$ sollte sich die Rechtswissenschaft ${ }^{9}$ indes jeglichen Gesetzespositivismus versagen - anders formuliert -, sich stets der historischen wie international-komparativen Dimension von Rechtsgeltung innewerden. 10

3 Vgl. dazu auch Becker, Rechtsvergleich und Rechtsdogmatik, in diesem Band, S. 11 ff.

4 Larenz/Canaris, Methodenlehre der Rechtswissenschaft, 3. Aufl. Berlin/Heidelberg/New York 1995, S. 17.

5 So Esser, Möglichkeiten und Grenzen des dogmatischen Denkens im modernen Zivilrecht, AcP 172 (1972), S. 98, 103.

6 Luhmann, Rechtssystem und Rechtsdogmatik, Stuttgart/Berlin/Köln/Mainz 1974, S. 15.

7 Jahr, Zum Verhältnis von Rechtstheorie und Rechtsdogmatik, in: ders./Maihofer (Hrsg.), Rechtstheorie - Beiträge zur Grundlagendiskussion, Frankfurt/Main 1971, S. 303.

8 Dazu Larenz/Canaris, Methodenlehre der Rechtswissenschaft (Fußn. 4), S. 263 ff.

9 Vgl. dazu auch Becker, Rechtsvergleich und Rechtsdogmatik, in diesem Band, S. 11 ff.

10 Vgl. dazu auch Larenz/Canaris, Methodenlehre der Rechtswissenschaft (Fußn. 4), S. 62 f. 
Am Klarsten formulierte die Aufgabe aller Rechtswissenschaft exemplarisch für das Zivilrecht Günther Jahr: ${ }^{11}$ „Soweit zivilrechtswissenschaftliche Bemühung über eine Dogmatik, Geschichte oder bloß äußerliche Vergleichung hinausgeht, sowie sie versucht, über das konkrete zivilrechtliche Phänomen des geltenden deutschen, eines früheren oder eines geltenden ausländischen Rechts hinauszugelangen und etwas über ,Zivilrecht überhaupt' auszusagen, ist sie nicht mehr nur Dogmatik, Geschichte oder Vergleichung, sondern zugleich Denken in einer anderen Dimension, in der auch die Beiträge anderer rechtswissenschaftlicher Disziplinen, vor allem der Rechtsphilosophie, der allgemeinen Rechtstheorie und der Rechtssoziologie, zu zivilrechtlichen Fragen zu bedenken sind, die man ,allgemeine Zivilrechtslehre' oder ,Zivilrechtstheorie' nennen könnte“. Denn ,,jede Bemühung um Verständnis zivilrechtlicher Phänomene eines historischen oder geltenden, inländischen oder ausländischen Rechts ist zugleich Bemühung um Verständnis von Zivilrecht überhaupt". 12

Was hier für das Zivilrecht formuliert ist, wäre für das Sozialrecht anzustreben, wohl wissend, dass diesem an jener Tradition und begrifflichen Durchbildung gebricht und auch dort die Skepsis an der Möglichkeit zu „wissenschaftlicher“ Durchbildung des Rechts als „System“ heute längst Legion geworden ist. ${ }^{13}$ Ist vielleicht die fehlende Systematisierbarkeit gar eine der „Eigenheiten“ des Sozialrechts?

Dogmatik verhält sich zum Recht wie die Grammatik zur Sprache. Beide sind gedankliche Abstraktionen, die zum korrekten Umgang mit Sprache und Recht verhelfen, namentlich deren Erlernbarkeit sichern sollen. Beide ermöglichen den Vergleich unterschiedlicher Sprachen und Rechte, weil ihre Begriffe für Verschiedenheiten offen sind und damit der Komparation die Grundlagen schaffen.

Dogmatische Instrumente haben sich am Recht wie grammatische Regeln an der Sprache zu bewähren, indem sie dem Recht Regelhaftes entnehmen. Sie sind tauglich, wenn sie Eigenheiten des Rechts begrifflich festhalten. Dogmatik und Grammatik gelten in der Zeit, weil sie das Recht oder die Sprache einer Zeit in ihrer Regelhaltigkeit darstellen. Wie sich Sprachgebrauch und Recht verändern, ändern sich auch Grammatik und Dogmatik.

Die Frage nach einer Dogmatik des Sozialrechts zielt auf dessen Leitideen und -prinzipien. Sie vermitteln und bezeichnen das Hintergrundwissen, das zur Deutung der positivrechtlichen Regeln nötig ist. Dogmatische Sätze stellen den normativen Gehalt normativer Institutionen fest. Dieser wird durch die Gesetzgebungsakte der einzelnen Rechtsordnung bestimmt und vorgegeben. Häufig folgt ein Gesetzgeber international verbreiteten Mustern; er befolgt sie nicht einheitlich, aber ähnlich. In der Welt der Wohlfahrtsstaatlichkeit bestehen unterschiedliche Muster, Modelle, Typen oder in der Sprache der zeitgenössischen vergleichenden Wohlfahrtsstaatsforschung formuliert:

11 Jahr, Romanische Beiträge zur modernen Zivilrechtswissenschaft, AcP 168 (1968), S. 9, 26.

12 Jahr (Fußn. 11), AcP 168 (1968), S. 25.

13 Meyer-Cording, Kann der Jurist heute noch Dogmatiker sein?, Tübingen 1973. 
„Welten des Wohlfahrtskapitalismus“. Sie taugen auch als Basis für eine dogmatische Theoriebildung, weil sie Bauprinzipien sozialrechtlichen Institutionen bestimmen.

\section{Der Wandel}

Die heutigen sozialen Strukturen der westlichen Gesellschaften weisen nur noch wenig Gemeinsamkeiten mit denen früherer Epochen auf. ${ }^{14}$ Die durchschnittliche Lebenserwartung der Menschen ist auf ein nie zuvor annähernd hohes Alter angestiegen; umgekehrt war die Geburtenrate seit Menschengedenken noch nie so niedrig wie gegenwärtig. Das durchschnittliche Lebensalter des homo sapiens steigt und - damit verbunden - wandelten sich seine Lebens- und Familienverhältnisse von Grund auf.

In dem Gedicht „Annchen von Tharau“ aus der Barockzeit erscheint die Ehe als das Urbild zwischenmenschlicher Solidarität. „Käm alles Wetter gleich auf uns zu schlahn / Wir sind gesinnet bei einander zu stahn. / Krankheit, Verfolgung, Betrübnis und Pein / Soll unsrer Liebe Verknotigung sein“. Die Ehe - einst als der Bund für das von Leiden geprägte Leben gedacht - ist heute einem nüchternen Verständnis einer auf wechselseitiger Sympathie beruhenden Konsumptionsgemeinschaft gewichen, welche bei Erlöschen der Sympathie als zerrüttet gilt und deshalb zu beenden ist. Die Familie hat darüber ihren hermetischen, nahezu überzeitlichen Charakter als Abfolge des einzelnen im Generationenverband verloren. Sie wurde darüber zur Kleinfamilie, wenn nicht gar in vielen, jedenfalls viel zu vielen Fällen zur Lebensgemeinschaft von Mutter und Kind ${ }^{15}$. Längst gehören wechselnde Lebenspartner im Lebenszyklus und in Familien zusammenlebende Kinder aus unterschiedlichen Lebensgemeinschaften der Eltern zum vertrauten Bild. Vieles von dem, was einst als gesicherte sittliche Grundlage des Rechts galt - die lebenslange Ehe oder die Familie mit womöglich vielen Kindern -, ist bestenfalls noch als höchst selten verwirklichtes Ideal anzutreffen, aber keineswegs mehr bestimmender Teil der gesellschaftlichen Wirklichkeit. Dieser Wandel hat auch das Recht zutiefst verändert - und zwar das Familienrecht ${ }^{16}$ ganz ebenso wie das familienfördernde Sozialrecht.

14 Goody, Geschichte der Familie, München 2002; Finch, Family Change, in: Bradshaw/Hatland (Hrsg.), Social Policy, Employment and Family Change in Comparative Perspective, Cheltenham 2006, S. 13; Dethloff, Gutachten 67. DJT (2008), A 12 ff.; Nave-Herz, Familie heute, Wandel der Familienstrukturen und Folgen für die Beziehung, Darmstadt 1994; Beck-Gernsheim, Die Kinderfrage heute: über Frauenleben, Geburtenrückgang und Kinderwunsch, München 2006; Statistisches Bundesamt, Familien in Deutschland - Ergebnisse des Mikrozensus 2006, Wiesbaden 28.11.2007; dass., Pressemitteilung Nr. 481 vom 28.11.2007.

15 Allerdings kein neues Phänomen, Goody, Geschichte der Familie (Fußn. 14), S. 229 ff.

16 Vgl. dazu eingehend Gernhuber/Coester-Waltjen, Familienrecht, 5. Aufl. München 2006, §§ 1-7. 


\section{Wandel des Familien- und familienfördernden Sozialrechts}

Das Familienrecht wurde zur rechtssystematischen Einheit des Privatrechts erst im 19. Jahrhundert - namentlich durch das BGB. ${ }^{17}$ Ältere Kodifikationen wie der code civil oder das ABGB ordneten es noch dem Personenrecht als unselbständiges Teilgebiet ein wie unter. Denn die Ehe und Familie als Unterhalts- und Konsumtionsgemeinschaft ging erst im ausgehenden 18. Jahrhundert aus der sich damals ebenfalls herausbildenden bürgerlichen Marktgesellschaft hervor. Diese war seitdem als Ort der Gütererzeugung und -verteilung vom Vertrag beherrscht, wogegen die Familie zum Ort gemeinsamer Konsumtion, von Intimität und Familienerziehung wurde und darin auf einem eigenen rechtlichen Status beruhte. Der damit bewirkten Spaltung der Lebenswelten in Familie und bürgerliche Gesellschaft entsprach die Ausdifferenzierung der Geschlechterrollen. ${ }^{18}$

Wurde die Gesellschaft zum Betätigungsort des Mannes, hatte die Frau als Hausfrau drinnen im Haus und Hof für Mann und Kinder zu walten. Auf dieser Unterscheidung zwischen Familie und bürgerlicher Gesellschaft beruhen BGB wie Sozialversicherungsrecht. Im ursprünglichen BGB war der Mann zur Erwerbsarbeit verpflichtet und stand dem Haushalt vor. Diesen hatte die Frau kraft Gesetzes nach dessen Weisungen zu führen; im Gegenzug schuldete er ihr Unterhalt. ${ }^{19}$ Dieses Modell fand im Sozialversicherungsrecht der frühen Jahre seine Entsprechung und Ergänzung. ${ }^{20}$ Die Witwenversorgung sollte die Frau schützen, falls der Mann verstorben war; Familienkrankenhilfe entlastete den Mann vom Vorsorgeunterhalt für die Frau.

Dieses einst patriarchalische Familienrecht ist in den vergangenen 50 Jahren durch ein partnerschaftliches Ehe- und Familienrecht abgelöst worden. ${ }^{21}$ Beide Ehegatten sind heute in Ehe und Familie wie Gesellschaft gleichberechtigt, voneinander unabhängig

17 Zweigert/Kötz, Einführung in die Rechtsvergleichung, Tübingen 1996, 3. Aufl. S. 150 ff.; Koch, Familie, Familienrecht, in: Handwörterbuch zur deutschen Rechtsgeschichte, 2. Aufl. Berlin 2007, 6. Lieferung.

18 Pateman, The patriarchal welfare state, in: Pierson/Castles (Hrsg.), The welfare state, 2. Aufl. Cambridge 2006, S. 134, 136.

19 Dies entsprach auch dem Common Law, vgl. Fredman, Discrimination Law, Oxford 2002, S. 27 : „Under the common law, ..., marriage constituted of a legal obliteration of women's identity”; hier schloss sich die feministische Kritik an: Pateman, in: Pierson/Castles (Hrsg.), The welfare state (Fußn. 18), S. 134 ff.: "The category of 'breadwinner' presupposes that wives are constructed as economic dependents or 'housewives', which places there in a subordinate position" (135); „The model of the couple as a financial unit bore little relation to reality as many women experienced it" (McIntosh, Feminism and Social Policy (1981), ebda., S. 120 ff.).

20 In der internationalen Diskussion als ,male breadwinner“-Model bezeichnet: Mayhey, The Parental Employment Context, in: Bradshaw/Hatland (Hrsg.), Social Policy (Fußn. 14), S. 37; Rust, Historische Entwicklung und gegenwärtige Bedeutung von Familienleistungen in der Sozialversicherung, VSSR 1996, S. 103.

21 Als Ausdruck der ,liberal-feminist agenda for a gender-neutral social world“ (Pateman, in: Pierson/Castles (Hrsg.), The welfare state (Fußn. 18), S. 134 ff.). 
und in der Rechtstellung selbständig. Beide haben ein Anrecht auf Teilhabe am gemeinschaftlich Erwirtschafteten. Die väterliche, später elterliche Gewalt über die minderjährigen Kinder wurde durch die elterliche Sorge abgelöst. Hinter dieser terminologischen Veränderung steht ein den eingetretenen rechtlichen Wandel auslösender wie befördernder gesellschaftlicher Wandel im Verständnis des Elternrechts. Dieses wird heute durch das Kindeswohl bestimmt und wandelte sich dabei vom Herrschaftsrecht zur Elternpflicht. ${ }^{22}$ Diese folgt aus dem pflichtgebundenen Elternrecht (Art. 6 II GG), der ein Recht des Kindes auf Erziehung gegenübersteht. Das Elternrecht formuliert heute ein Leistungsprogramm in einem von gegenseitigen Rechten und Pflichten geprägten Schuldverhältnis. Kinderrechte ${ }^{23}$ sind heute - freilich erst seit kurzem - der unverrückbare Ausgangspunkt zur Bestimmung der elterlichen Rolle. Die rechtspraktischen Folgen waren und sind erheblich. Aus den Kinderrechten erklärt sich beispielsweise die Rechte auf Kenntnis der eigenen Abstammung 24 und des Umgangs des Kindes mit jedem Elternteil. ${ }^{25}$ Es verwirklicht das Recht jedes Kindes auf beide Eltern ${ }^{26}$ und wertet die Stellung des biologischen Vaters gegenüber dem rechtlichen Vater auf, ja macht sie als rechtseigene Stellung erst eigentlich begründbar. ${ }^{27}$

Wird die Elternstellung aber von den Kindesrechten her gedeutet, verliert ferner die überkommene Unterscheidung der Kinder nach ihrer Abstammung jegliche Bedeutung. Denn unter den Bedingungen zeitgenössischer Reproduktionsmedizin ist Abstammung eindeutig zu klären; es bedarf also nicht mehr des Rückgriffs auf sinnfällige Ausdrucksformen zwischenmenschlicher Nähe.

Neben die Ehe sind inzwischen rechtlich anerkannte gleichgeschlechtliche Lebenspartnerschaften ${ }^{28}$ getreten: Ihnen wird mitunter das Recht zur Familiengründung eröffnet. Ein Diskriminierungsverbot im Hinblick auf die sexuelle Identität brandmarkt

22 BVerfG - 1.4.2008 - FamRZ 2008, S. 845: „Die den Eltern durch Art. 6 II 1 GG auferlegte Pflicht zur Pflege und Erziehung ihres Kindes besteht nicht allein dem Staat, sondern auch ihrem Kind gegenüber. Mit dieser elterlichen Pflicht korrespondiert das Recht des Kindes auf Pflege und Erziehung durch seine Eltern aus Art. 6 II 1 GG. Recht und Pflicht sind vom Gesetzgeber auszugestalten.“ Verbot der Gewalt (§ 1631 II BGB), vgl. Coester, Elterliche Gewalt, in: Hofer/Klippel/Walter (Hrsg.), Perspektiven des Familienrechts, Festschrift für Dieter Schwab, Bielefeld 2005, S. 747.

23 Zacher, Universale Menschenrechte und die Wirklichkeit der globalen Welt, ZIAS 2007, S. 66 ff.; zum Verhältnis Eltern-Kinderrechte, ders., Elternrecht, in: Isensee/Kirchhof (Hrsg.), Handbuch des Staatsrechts der Bundesrepublik Deutschland, Band VI, Heidelberg 2001, § 134-69. Es wird zu Recht ein „Konflikt zwischen Elternrecht und Kindesgrundrechten“ ausgemacht, und zu Recht betont, dass das Elternrecht im Dienst der Kindesgrundrechte steht - nämlich als Ergänzung und Mittel zu deren Verwirklichung.

24 BVerfGE 79, 256; 90, 263; Schwab, Familienrecht, 15. Aufl. München 2007, Rdnr. 492-495.

25 BVerfG, FamRZ 2007, S. 105; EGMR, FamRZ 2001, S. 341; 2002, S. 381.

26 Ebert, Die Defizite im deutschen Kindschaftsrecht, in: Koeppel (Hrsg.), Kindschaftsrecht und Völkerrecht, Neuwied/Kriftel/Berlin 1996, S. 103, 111.

27 Büttner, Der biologische (genetische) Vater und seine Rechte, in: Hofer/Klippel/Walter (Hrsg.), Perspektiven des Familienrechts (Fußn. 22), S. 735.

$28 \mathrm{Vgl}$. Schwab, Familienrecht (Fußn. 24), §§ 94 ff. 
heute vieles als Zurücksetzung, was noch vor wenigen Jahrzehnten als naturgegebener Wesensunterschied erschien und daher rechtlich gebilligt wurde. ${ }^{29}$

\section{Familien- und Sozialrecht im Zeichen der Gleichbehandlung der Ge- schlechter}

Ein den Geschlechtern verschiedene Aufgaben in Erwerbsleben und Familie zuweisendes Familien- und Sozialrecht ${ }^{30}$ wurde so in den vergangenen Jahrzehnten weithin überwunden. ${ }^{31}$ Gegenwärtig noch vorhandene und fortbestehende Benachteiligungen von Frauen oder Müttern gegenüber Männern und Vätern ${ }^{32}$ sind als unmittelbare und mittelbare Diskriminierung prinzipiell geächtet. Sie können daher bei konkretem Aufund Nachweis rechtlich unterbunden werden. In Folge solchen Wandels sind die einst vorherrschenden, an das Geschlecht gebundenen Rechte und Pflichten durch deren geschlechtsneutrale Ausgestaltung verdrängt und ersetzt worden. Dies erwies sich als folgenreich - sowohl für das Familien- wie das Sozialrecht.

Die erste, für Familien- wie Sozialrecht gleichermaßen bedeutsame Folgerung dieses Wandels ist die Preisgabe jeglicher Rollen- und Aufgabenzuweisung für Männer und Frauen in Ehe und Familie. ${ }^{33}$ Damit stehen Mann und Frau der Zugang zu Erwerbsund Familienarbeit unter den gleichen rechtlichen Bedingungen und Voraussetzungen offen. Für das Familienrecht bedeutete dies, die Aufgabenzuweisung in der Ehe den Eheleuten zu überlassen; für das Sozialrecht folgte daraus, dass die Familienkrankenhilfe auf den nichterwerbstätigen Ehegatten beschränkt und die Hinterbliebenenrente geschlechtsneutral formuliert wurde. Infolgedessen wurden die vormals differenzierten Anspruchsvoraussetzungen für die Witwen- und Witwerrenten überwunden und einander vollständig angeglichen.

Die geschlechtsneutrale Ausgestaltung sozialer Rechte führte zu deren Erweiterung auf bislang Nicht-Geschützte: Witwer und nicht oder eingeschränkt erwerbstätige Ehemänner. Sie begünstigte ferner die Aufteilung sozialer Rechte, was sich namentlich im

29 Ausschluss der Lebenspartner auf die berufsständische Versorgungswerke: EuGH - 1.4.2008 - C267/06 (Maraku); anders noch BGH - 14.2.2007 - IV ZR 267/04 = FamRZ 2007, S. 805, im Hinblick auf Betriebsrenten.

30 Vgl. Überblick: Eichenhofer, Sozialrecht, 6. Aufl. Tübingen 2007, Rdnr. 145.

31 Schwenzer, Ein Familienrecht für das 21. Jahrhundert, in: Deutscher Familiengerichtstag e.V. (Hrsg.), Brühler Schriften zum Familienrecht, 17. Deutscher Familiengerichtstag, Bielefeld 2008.

32 Vgl. Scheiwe (Hrsg.), Soziale Sicherungsmodelle revisited, Baden-Baden 2007; Sainsbury, Gender, Equality and Welfare States, Cambride 1996.

33 Ruland, Familiärer Unterhalt und Leistungen sozialer Sicherheit, Berlin 1973; Deutscher Sozialrechtsverband, Die Familie im Sozialleistungssystem, Berlin 2008. 
Versorgungsausgleich ${ }^{34}$ wie Rentensplitting zeigt. Auf diese Weise können die auf Einkommensunterschieden zwischen den Eheleuten während der Dauer der Ehe bewirkten Ungleichheiten im sozialen Schutz jedenfalls ausgeglichen und damit überwunden werden.

Diese Umwälzungen dürfen nicht als Kulturrevolution oder Fehlentwicklungen eines - prinzipiell oder konkret - als gering zu schätzenden Zeitgeistes missverstanden werden. Sie geben vielmehr einem tiefgreifenden Wandel der Lebensverhältnisse ihren rechtlichen Ausdruck, welcher gerade auch die Beziehungen unter den Geschlechtern und das Verhältnis von Eltern und Kindern verändert hat. Die Technisierung von Hausund Erwerbsarbeit und die Ablösung der Industrie- durch die Dienstleistungsgesellschaft $^{35}$ verbunden mit einer Universalisierung der Menschenrechte - der auch die Vorstellung vorgeblich naturgegebener Rechts- und Rangunterschiede zwischen Männern und Frauen zuwiderlief - verlangte nach tiefgreifenden rechtlichen Reformen, die zu der neuen wirtschaftlichen, sozialen und kulturellen Wirklichkeit passten. Auch das deutsche familienfördernde Sozialrecht hat sich auf diesen Wandel eingelassen - mit rapidem Fortgang dieses Prozesses.

Diese Änderungen lassen sich auch keineswegs als deutsche Spezialitäten oder gar Kuriositäten abtun, sondern entsprechen dem EU-Standard, der sich inzwischen auch in der Familienpolitik herauszubilden beginnt. ${ }^{36}$ In allen europäischen Staaten ändert sich gegenwärtig das Verhältnis von Eigen-, Familien- und Solidarverantwortung tiefgreifend. Einerseits werden durch Reformen im Unterhalts- und Arbeitsförderungs-, aber auch Rentenversicherungsrecht im Zeichen des aktivierenden Sozialstaates die Anforderungen an die Eigensorge- und -vorsorge der arbeitsfähigen Menschen erhöht, um so die Familien- und Solidarverantwortung zu senken. Andererseits steigern die europäischen

34 Vgl. dazu eingehend vergleichend Reinhard, Rechtsordnungen mit Versorgungsausgleich im Sinne des Art. 17 Abs. 3 EGBGB, Baden-Baden 1995.

35 OECD, Babies and Bosses, Reconciling Work and Family Life, A synthesis of Findings for OECD Countries, Paris 2007, S. 42.

36 Kommission der Europäischen Gemeinschaft vom 16. März 2005, Angesichts des demographischen Wandels - eine neue Solidarität zwischen den Generationen, KOM (2005), 94 endg.; dazu auch Max-Planck-Institut für ausländisches und internationales Sozialrecht, Eigen-, Familien- und Solidarverantwortung im Sozialrecht (im Erscheinen); European Commission, Directorate-General for Employment, Social Affairs and Equal Opportunities, Joint Report on Social Protection and Social Inclusion 2008. Social Inclusion, pensions, health care and long term care. Die Priorität der Sozialpolitik der EU müsse auf der schnellen und nennenswerten Verminderung der Kinderarmut liegen (S. 6); weiter: ,the labour market situation of parents is a key determinant of the conditions in which children live and develop. Earnings from work are normally the main source of income for parents in their prime age, and joblessness represents the main risk of poverty, for households with children" (S. 11); vgl. auch die Sicht der IAO Gillion/Turner/Bailey/Latulippe, Social Security Pensions. Development and Reform, ILO, Genf 2008, S. 397, mit der Beobachtung, dass die auf den „breadwinner-Typus” zugeschnittenen und ausgerichteten Rentensysteme „did not forsee the expansion of female employment and the subsequent increase in the proportion of women with their own entitlement to a pension. This turned out to be inconsistent with the earlier picture of women's pension being derived from that of the male wage earner". 
Gesellschaften ihre Anstrengungen, um den Einzelnen zur eigenverantwortlichen Wahrnehmung von Familienverantwortung unter den Bedingungen der sich zur Dienstleistungsgesellschaft gewandelten Erwerbsgesellschaft zu bewegen.

\section{Familienförderung - Eheförderung}

Der oftmals behauptete - in des Wortes ursprünglichem wie übertragenem Sinn „genetische“ Zusammenhang zwischen Ehe und Familienförderung war im deutschen Sozialrecht freilich nie so eng, wie es dessen rechtspolitische Kritiker glauben machen wollten. Gewiss waren einst Hinterbliebenenversorgung und Familienkrankenhilfe den Ehefrauen vorbehalten; jedoch wurden seit jeher beide Leistungen den ehelichen wie nichtehelichen, angenommenen wie Pflege- und Stiefkindern zuteil. Dem Sozialrecht lag daher schon seit langem das auf faktischer Elternstellung beruhende zeitgenössische Familienverständnis zugrunde. Daher ist die These, die Familienförderung des deutschen Sozialrechts leiste einen Beitrag zur Stärkung einer antiquierten und traditionellen Ehemoral, nie berechtigt gewesen. Im Gegenteil, im zeitgenössischen Konzept der Bedarfsgemeinschaft wird deutlich, dass privates Einstehenmüssen gerade nicht an die Ehe gebunden ist.

Der sozialrechtliche Schutz der Ehe geschah also nicht um der Eheförderung willen, sondern erklärte sich aus der familienrechtlich geforderten Solidarität unter Eheleuten, die durch die genannten Sozialleistungen ergänzt und vervollständigt wurde. Diese begründete den Vorrang der Familie vor dem Sozialstaat bei der Bedarfssicherung. Auf ihr baut vor allem auch das bedürftigkeitsabhängige Sozialrecht auf, indem es - getreu dem Subsidiaritätsprinzip - den bedürftigen Ehegatten vorrangig auf den unterhaltspflichtigen Ehegatten statt den Sozialhilfeträger verweist. Hat die familienrechtliche Unterhaltspflicht aber gegenüber allen bedürftigkeitsabhängigen Sozialleistungen den Vorrang, liegt es umgekehrt nahe, den krankheits- oder todesbedingten Verlust des Unterhalts zum eigenen Leistungsfall in Sozialversicherung und sozialer Entschädigung zu vertypen. Gilt die Ehe in allen europäischen Staaten unabhängig vom vorherrschenden Typus der Wohlfahrtsstaatlichkeit als Bedarfsgemeinschaft, deren Mitglieder gegenüber der Allgemeinheit vorrangig zur Bedarfsdeckung Bedürftiger verpflichtet sind, erscheint es nur folgerichtig, auch im Unterhaltsverlust einem ausgleichspflichtigen Sozialleistungstatbestand zu sehen.

Die nach dem Subsidiaritätsgrundsatz entwickelte Zuständigkeitsverteilung zwischen der Solidar- und Familienverantwortung verliert jedoch - näher betrachtet - an Klarheit und Strahlkraft und zwar aus ökonomischen Gründen. Reicht die unterhaltsrechtlich erhebliche Leistungsfähigkeit des Unterhaltsschuldners nicht hin, die Bedarfe des Unterhaltsgläubigers zu decken - namentlich in den häufigen Mangellagen, dass mehrere Unterhaltsgläubiger einem leistungsschwachen Unterhaltsschuldner gegenüber stehen - 
wird der regelmäßig seitens der Unterhaltsgläubiger unbefriedigte Bedarf wegen der hohen Kosten für die Durchsetzung oftmals durch die mit weit geringeren Transaktionskosten und Beitreibungsrisiken verbundene Inanspruchnahme von Sozialhilfeleistungen im Rahmen von Sozialhilfe, der Grundsicherung und beim Unterhaltsvorschuss ersetzt. Die Träger der Sozialhilfe sind dann zwar zum Rückgriff befugt; dieser wird aber durch den aus Gründen sozialen Schutzes für Unterhaltsschuldner geltenden Pfändungsschutz ${ }^{37}$ erschwert. Daraus können die durch Sozialrecht auszugleichenden Funktionsausfälle privaten Unterhaltsrechts erwachsen, welche die Subsidiarität des Sozialrechts insgesamt in Frage stellen. Wie beispielhaft und -gebend im Unterhaltsvorschussrecht erkennbar, droht der Sozialstaat aus seiner angestammten Stellung nachrangiger zum erstrangigen Unterhaltsschuldner zu werden. Die Primärzuständigkeit des Familienrechts und die subsidiäre Auffangzuständigkeit des Sozialhilferechts scheinen sich so zu verkehren.

Nichteheliche Lebensgemeinschaften sind in Sozialhilfe, Grundsicherung und Wohngeldrecht zwar als Bedarfsgemeinschaften anerkannt, aber zugleich nicht in die abgeleiteten Sicherungen des Sozialversicherungs- und sozialen Entschädigungsrechts einbezogen. Der gelegentlich vernommene Vorwurf einer Ungleichbehandlung erweist sich jedoch als unbegründet. Denn die vom Gesetz unterstelle faktische Unterhaltsgewährung unter den Mitgliedern der Bedarfsgemeinschaft rechtfertigt sich daraus, dass eine sittlich begründete Unterhaltsleistung die nachträgliche Kondiktion des Erbrachten ausschließt (§ 814 BGB). Aus ihr erwachsen aber keine Ansprüche auf Unterhaltsgewährung für die Zukunft, die einzig Grundlage einer sozial(versicherungs)rechtlichen Absicherung werden könnten. 38

Weit weniger klar begründet erscheint dagegen die Gleichstellung von Ehe, Lebenspartnerschaft und eheähnlicher Gemeinschaft bei Gewährung von sozialrechtlichen Vorteilen, die historisch zunächst für Eheleute geschaffen wurden. Es hängt vom Familienrecht des einzelnen Staates ab, inwieweit die Lebenspartnerschaft der Ehe gleichgestellt ist. ${ }^{39}$ Hier bestehen nach wie vor Unterschiede. Darüber hinaus gibt es unterschiedliche Vorstellungen darüber, inwieweit die eheähnliche Gemeinschaft der Ehe gleichzustellen ist.

$37 \S 850$ c ZPO Arbeitseinkommen von $930 €$ monatlich, für Unterhaltspflichtige von $2.060 €$ sind unpfändbar; vgl. eingehend Martiny, Unterhaltsrang und -rückgriff, Bd. 2, Tübingen 2000, S. 1090 ff.; Dethloff, 67. DJT (2008), S. $122 \mathrm{ff}$.

38 Allerdings besteht in vielen Staaten die neuere Tendenz, für faktische Lebensgemeinschaften gesetzliche Ausgleichsregelungen zu schaffen: vgl. Dethloff, Unterhalt, Zugewinn, Versorgungsausgleich - Sind unsere familienrechtlichen Ausgleichssysteme noch zeitgemäß?, Verh. 67. DJT (2008), A 35 ff.

39 Vgl. hierzu den Einfluss der Rechtsprechung des EuGHMR v. 21.12.1999 - Salgueirs de Silva Monta v. Portugal: die Weigerung der Bevormundung eines Kindes durch homosexuellen Vater verletze dessen Recht auf Familienleben (Art. 8 EMRK) und bedeute eine Geschlechterdiskriminierung (Art. 14 EMRK). 
Dabei kollidieren zwei einander widerstreitende, rechtlich gleichermaßen anerkannte Prinzipien. Einerseits fordert der Schutz von Ehe und Familie, dass deren Privilegierung einen exklusiven Charakter haben müsse; andererseits kann jedoch heute von jedermann auch ein Schutz vor der Diskriminierung wegen des familienrechtlichen Status ${ }^{40}$ oder der sexuellen Identität in Anspruch genommen werden. Daraus kann die Folgerung abgeleitet werden, dass der Ehe gegenüber eheähnlichen Gemeinschaften und Lebenspartnerschaften kein Vorrang zukommen darf. Der Streit um die Vorherrschaft der beiden rivalisierenden und miteinander unverträglichen Prinzipien hält noch an und dessen Lösung ist noch nicht endgültig abzusehen. ${ }^{41} \mathrm{Je}$ nachdem, worauf die einzelne Rechtsordnung ihren Akzent legt, wird sie deshalb dem einen oder anderen Prinzip den Vorrang einräumen.

\section{Familienförderung - neues Aufgabenfeld des Sozialrechts}

Weil die Überwindung der Geschlechterrollen in Familien- und Sozialrecht ihre Entsprechung in dem letztlich gemeinschaftsrechtlich formulierten und fundierten Gebot der Gleichbehandlung der Geschlechter im Arbeits- und Erwerbsleben gefunden hatte ${ }^{42}$ (Artt. 141, 3 II EG), folgte aus der Gesamtheit dieser Angleichbestrebungen auch eine Zunahme der Erwerbsbeteiligung der Frauen. Dies führte trotz fortbestehender nicht zu leugnender Ungleichheiten insgesamt zur Annäherung der Erwerbsbiographien von Männern und Frauen. Die Veränderung hin zu einer wissens-, informations- und kommunikationsbasierten Wirtschaft kommt den hergebracht als „,weiblich“ geltenden Befähigungen sogar entgegen, werden diese damit für den Arbeitsmarkt doch zunehmend zentral. 43

Freilich bedeutet eine im Zeichen der Gleichbehandlung der Geschlechter stehendes Familien- und Sozialrecht zunächst nur ein normatives Ideal, dem die gesellschaftliche Wirklichkeit noch nicht entspricht. Nach wie vor sind Löhne, Renten, Aufstiegschancen, Arbeitsplatz- und Prekarisierungsrisiken unter Männern und Frauen ungleich verteilt, weshalb auch künftig kompensatorische Maßnahmen geboten wie begründet

40 Namentlich anerkannt in der Rechtsprechung des EuGHMR - Rs. Wessels-Bergervoot - 4.6.2002 Application no. 34462/97; stellt die Eheschließung ein Freiheitsrecht dar, ist auch dessen Nichtgebrauch geschützt; möglich ist auch Postulat aus dem Verbot einer Diskriminierung auf Grund des Versorgerstatus abzuleiten: Ketscher, Sozialret, 3. Aufl. Kopenhagen 2008, S. 272.

41 Dethloff, Gutachten, 67. DJT (2008), A 134 ff., A 144 ff., plädiert für Ausgleichsregelungen für „,bestimmte dauerhafte oder verfestigte Lebensgemeinschaften“ einen Anspruch auf Ausgleich wirtschaftlicher Ungleichgewichtslagen.

42 Vgl. Streinz-Eichenhofer, EGV, EUV, München 2003, Art. 141 Rdnr. 13 ff.

43 OECD, Babies and Bosses (Fußn. 35), S. 42: „By 2005, two thirds of all OECD employment was in the service sector, and with four one of five women in service sector employment, the importance of this sector's growth in fostering female labour participation can hardly be exaggerated." 
sind. ${ }^{44}$ Aber es gibt im Hinblick auf die Gleichstellung Differenzen zwischen den europäischen Staaten, die nicht aus Unterschieden in Familien- und Sozialrecht rühren, sondern sozial und kulturell bedingt sind. Sie haben insbesondere Unterschiede in weiblichen und männlichen Lebensentwürfen zur Folge. Dies zeigt: Ähnliches Recht wirkt in sozial und kulturell unterschiedlichen Gesellschaften unterschiedlich.

Jede auf Gleichstellung von Frauen und Männern im Erwerbsleben gerichtete Politik bejaht implizit die Erwerbsbeteiligung der Frauen. ${ }^{45}$ Im Hinblick auf die Grundrechte der EU ist die Vereinbarkeit von Erwerbs- und Familienarbeit ein neues und eigenständiges soziales Grundrecht (Art. 33 Charta der EU-Grundrechte). ${ }^{46}$ Jede Gleichstellungspolitik steht deshalb vor der Frage, wie Familiengründung und -erweiterung ermöglicht werden könnten. ${ }^{47}$ Diese Frage enthüllt spezifische und beträchtliche Benachteiligungsgefahren für Frauen. Die gegenwärtig europaweit beobachtbaren Bestrebungen zur Ausweitung der Familienleistungen gehören in diesen Zusammenhang.

Die sozialrechtliche Familienförderung hat zunächst bescheiden im Jugendwohlfahrts- und Kindergeldrecht begonnen. Erstes war anfangs darauf beschränkt, das staatliche Wächteramt über die Wahrnehmung des Elternrechts als Elternpflicht auszuformen. Kindergeld sollte das elementare, einst bereits in der Sozialenzyklika Rerum Novarum (1891) niedergelegte Postulat der katholischen Soziallehre nach einer familiengerechten und bedarfsdeckenden Entlohnung 48 verwirklichen. Insoweit ist es als soziales Grundrecht inzwischen weltweit anerkannt. Dessen Verwirklichung konnte durch das Arbeitsrecht - also internalisierend - nicht gelingen und musste daher im Hinblick auf den Schutz der Kinder sozialrechtlich, also externalisierend ${ }^{49}$ gewährleistet werden.

Neben diese Maßnahmen tritt heute europaweit ein neues Instrumentarium der sozialrechtlichen Ermöglichung und Begleitung von Elternschaft. Eigenverantwortung bedeutet im Familien- und Sozialrecht zunächst und vor allem, dass die eigene Erwerbstätigkeit dem Bezug von Familienunterhalt oder Sozialleistungen grundsätzlich vorgehen muss. Dies wird im skandinavischen Wohlfahrtsstaat ${ }^{50}$ explizit und am klarsten als Zielvorstellung formuliert, liegt als Prämisse aber auch den anderen Modellen des Wohlfahrtsstaats zugrunde. In Frankreich ${ }^{51}$ besteht die Pflicht zur Arbeit für den Ein-

44 OECD, Employment Outlook 2008, Paris 2008, S. 129 ff.; Stahlberg, Gender and Social Security: Some Lessons from Europe, 4 (2002) EU Journal of Social Security, S. 227.

$45 K \ddot{b} b l$, Frau und Beruf, 3. Aufl. München 1995, S. 21 ff.

46 Nußberger, in: Tettinger (Hrsg.), Kölner Kommentar zur Europäischen Grundrechte-Charta, München 2006, Erl. zu Art. 33.

47 Esping-Andersen, Social Foundations of Postindustrial Economies, Oxford 1999, S. 145, 167, 174 ff.

48 Enzyklika Rerum Novarum vom 15. Mai 1891, Tz. 34 f., in: Kerber/Ertl/Heinz, Katholische Gesellschaftslehre im Überblick, Frankfurt/Main 1991, S. 41, 44.

49 Zacher, Zur Anatomie des Sozialrechts, SGb 1982, S. 329.

50 Vgl. dazu Esping-Andersen (Fußn. 47), S. 167 ff..; Mayhew, The Parental Employment Context, in: Bradshaw/Hatland (Hrsg.), Social Policy (Fußn. 14), S. 37 ff.; Ketscher (Fußn. 40), S. 79 ff., 274 f.

51 Laborde/Monéger, Prestation familiales et contrôle social, No. 4, 1994, Revue de droit sanitaire et social; Kessler, Droit la protection sociale, 2. Aufl. Paris 2005, S. 280-299. 
zelnen und die dieser korrespondierenden Pflicht zur Schaffung von Arbeitsplätzen für den Staat! Deswegen wird es zu einem zentralen Anliegen und gemeinsamen Ziel von Familien- und Sozialrecht, durch Ausbildungsunterhalt, Rehabilitation, Teilhabe, Arbeitsvermittlung oder Berufsförderung die Befähigung jedes Einzelnen zur Erwerbsausübung und -beteiligung zu begründen, aufrechtzuerhalten oder wiederherzustellen.

Familien- wie Sozialrecht sind dabei von der sie beide verbindenden Grundannahme beherrscht und geleitet, dass durch eigenverantwortete Erwerbsarbeit die Daseinssicherung ohne fremde, familiäre oder öffentliche Hilfe möglich werde. Damit bekräftigen das zeitgenössische Familien- und Sozialrecht ein ihnen gemeinsam zugrunde liegendes Prinzip des Vorrangs der Selbstverantwortung gegenüber der Einstandspflicht Dritter. Diese Maxime bestimmt nicht nur das Rangverhältnis zwischen den beiden Rechtsgebieten, sondern gewinnt auch Bedeutung für die Ausrichtung der sozial- und familienrechtlichen Leistungen. Diese sollen Beschäftigung fördern und entfalten und nicht deren Aufnahme oder Ausweitung entgegenstehen.

\section{Sozialrechtliche Ermöglichung und Begleitung von Elternschaft}

Zeitgenössisches Sozialrecht ist angesichts dessen bemüht und praktisch darauf ausgerichtet, durch die umfassende wie kostenträchtige Mobilisierung von Solidarverantwortung dem Einzelnen die eigenverantwortliche Wahrnehmung seiner Familienverantwortung zu ermöglichen, ja die Eltern hierzu oft erst eigentlich zu befähigen. ${ }^{52} \mathrm{Sie}$ steht damit im Einklang mit den auch in der Arbeitsförderung 53 beobachtbaren europaweiten Versuchen, den Sozialstaat als aktivierenden und investiven Sozialstaat zu gestalten. ${ }^{54}$ Im Kern wird der soziale Staat statt als dauerhaft gewährender als befristet leistender und dadurch befähigender Staat gedacht. Dieses Konzept wird oftmals fälsch-

52 Eichenhofer, Sozialrecht (Fußn. 30), Rdnr. 497 ff., 486 ff.; Seiler, Grundzüge eines öffentlichen Familienrechts, Tübingen 2008, S. 51 ff.; Botsch, Eine Familienpolitik für junge Familien, NDV 2004, S. 210; Kaufmann, Herausforderungen des Sozialstaates, Frankfurt 1997, S. 69 ff., 168 ff.; Esping-Andersen, Social Foundations of Postindustrial Economies (Fußn. 47), S. 179: „A fundamental postindustrial dilemma - generally overlooked in contemporary debates - is that families such no longer indined to assume the costs of bearing children".

53 Vgl. dazu in diesem Band: Bieback, „Social assistance“ in Deutschland, Frankreich und im Vereinigten Königreich - von den ,,armen Armen“ zu den „berechtigten Armen“, S. 217 ff.; Igl, Soziale Hilfe und soziale Förderung in Frankreich im Rahmen der ,,action social“, S. 265 ff.; Schulte, „Fördern und Fordern“ - Aktivierung im Arbeitsmarkt in Deutschland und in Großbritannien, S. 273 ff.

54 Grundlegend: Gilbert, Welfare Justice: Restoring Social Equity, Yale 1995; ders., Remodeling Social Welfare, issue July/August 1998, 8 Society; Goodin, Reasons for welfare. The Political Theory of the Welfare State, Princeton 1988; Jordan, The New Politics of Welfare, London 1998. 
lich als ,neo-liberal“ apostrophiert; 55 tatsächlich beruht es jedoch auf einem kommunitaristischem Grundansatz. ${ }^{56}$

Diese Mobilisierung geschieht durch das und einkommensproportional bemessene und anders als in Schweden, wo das Modell entwickelt wurde, statt beitrags-, steuerfinanzierte Elterngeld, das Kindergeld, ${ }^{57}$ ferner durch Arbeits-, Kündigungs- und Entgeltschutz bei Mutterschaft sowie Rechtsansprüche auf öffentliche Kinderbetreuung. Nicht wenige der Regeln wurden durch das Europarecht verbreitet. Es unterscheiden sich innerhalb der verschiedenen Staaten Europas die Familienförderungen zwar noch in Ausrichtung, Akzentsetzung, Sicherungsniveau, Laufzeit und auch der Geschlechterbeteiligung, jedoch nicht mehr in der familien- und sozialpolitischen Grundtendenz. ${ }^{58}$ Die genannten Maßnahmen sind die Antwort der Politik auf ein neues soziales Risiko. 59 Unabhängig von der politischen Grundausrichtung eines Sozialleistungssystems häufen sich deswegen die Bemühungen der einzelnen Staaten durch den Ausbau sozialpolitischer Leistungen und Maßnahmen, den Familien bessere Lebens- und Entfaltungsbedingungen zuteil werden zu lassen. ${ }^{60}$ Der Mutterschutz für Arbeitnehmerinnen und Selbständige ist weitgehend europäisiert; desgleichen sind Elternzeiten - als Unterbrechungstatbestände für die abhängige Erwerbsarbeit wegen der Erziehung Neugeborener und Kleinkinder 61 - begründet worden. ${ }^{62}$ Sie werden regelmäßig durch Elterngeldzah-

55 Vgl. dazu Zintl, Die libertäre Sozialstaatskritik bei von Hayek, Buchanan und Nozick, in: Kersting (Hrsg.), Politische Philosophie des Sozialstaats, Weilerswist 2000, S. 95 ff.; Lessenich, Die Neuerfindung des Sozialen, Bielefeld 2008.

56 Vgl. dazu Reese-Schäfer, Kommunitaristisches Sozialstaatsdenken, in: Siegfried Blaschke/Dieter Döring (Hrsg.), Sozialpolitik und Gerechtigkeit, Frankfurt 1998, S. 75; grundlegend Etzioni, Die Verantwortungsgesellschaft, München 1997.

57 Felix, in: Ruland/von Maydell/Becker, SRH, 4. Aufl. Baden-Baden 2008, Tz. 30-60 ff., 70 ff.; Wangen, Der Familienlastenausgleich, Diss., Trier 2003.

58 Das Neue dieser Regelungen zeigt sich daran, dass sie sich nur schwerlich in das Koordinierungsrecht einfügen, vgl. Paskalia, Free Movement, Social Security and Gender in the EU, Hart Publishing, Oxford 2007, S. 7 ff.

59 Bonoli, The Politics of the New Social Policies: providing coverage against new social risks in mature welfare states, in Vol. 33 (2005) issue 3, Policy and Politics, S. 431-459.

60 Vgl. dazu für Bieback, Family Benefits: The New Legal Structures of Subsidizing the Family, 1992 (4) Journal of European Social Policy, S. 239-254; BMFSFJ, Familienbezogene Leistungen und Maßnahmen des Staates, 2006; Seiler, Grundzüge eines öffentlichen Familienrechts, Tübingen 2008, S. 106 ff.; Esping-Anderson, A New Gender Contract, in: ders., Why we need a New Welfare State, 2002, p. 68-70; OECD, Babies and Bosses (Fußn. 35); OECD, Employment Outlook 2008, S. $138 \mathrm{ff}$.

61 Kaufmann, Herausforderungen des Sozialstaates (Fußn. 52), S. 168.

62 RL 96/34/EG vom 3. Juni 1996, ABl. EG L 145 (Elternzeit) vom 19.6.1996, S. 4, RL 92/85/EWG vom 19. Oktober 1992 (Mutterschutz) ABl. EG L 348 vom 28.11.1992, S. 1; Communication from the Commission to the Council, the European Parliament, the European Economic and Social committee and the Committee of the Regions - A Roadmap for equality between women and men 20062010 (COM/2006/92 final); European Commission, Child Poverty and Well-Being in the EU, Januar 2008. 
lungen flankiert, ${ }^{63}$ die entweder als Lohnersatzleistungen oder öffentliche Bezuschussung von Kinderbetreuung von Säuglingen und Kleinkindern nach standardisiertem, vom Einkommen der Eltern abhängigen oder unabhängigen Sätzen bemessen sind.

Gewichtige Unterschiede werden jedoch namentlich im Hinblick auf die Kinderbetreuung sichtbar. ${ }^{64}$ Hier stehen sich die nordischen Staaten und Frankreich mit ihren stark ausgebauten Einrichtungen öffentlicher Kinderbetreuung einerseits, Italien mit einer nach wie vor starken Familienorientierung und das Vereinigte Königreich mit einer die Eltern wesentlich auf Privatrecht und Privatwirtschaft verweisenden Kinderbetreuung gegenüber. Deutschland nimmt in dieser Hinsicht nach wie vor eine höchst differenzierte Position ein. In der rechtspolitischen Diskussion erlangt der Betreuungsunterhalt eine wachsende Bedeutung. ${ }^{65}$ Dies deutet darauf, dass Kinderbetreuung einer Erwerbsarbeit gleichsteht. In manchen Gegenden, namentlich im Osten, rückt die Betreuungsinfrastruktur im Gefolge der DDR-Tradition in die Nähe der nordischen Staaten und Frankreichs, wogegen andernorts Anklänge an das britische und italienische Modell erkennbar sind. Je stärker die Kinderbetreuung als originäre Aufgabe der Familie betrachtet wird, desto mehr rückt die Frage ihrer sozialrechtlichen Flankierung, namentlich im Rentenrecht ins Blickfeld. ${ }^{66}$

Dessen ungeachtet besteht in allen Staaten, die noch Defizite zeigen, das Bestreben, einen höheren Grad an öffentlicher Kinderbetreuung in der Zukunft durchzusetzen. Mögen sich deshalb auch die Muster des Schutzes in den verschiedenen europäischen Staaten in wichtigen und der vergleichenden Würdigung zugänglichen Einzelheiten (Dauer, Höhe, Laufzeit, Geschlechterbeteiligung) unterscheiden 67 , besteht in der familien- und sozialpolitischen Grundtendenz dennoch eine ganz auffällige Übereinstimmung. Diese gestalterische Vielfalt erweist sich als Variationen auf ein und dasselbe Thema.

Das Recht der Jugendhilfe durchläuft in diesem Zusammenhang einen elementaren Transformationsprozess seines rechtssystematischen Status - wandelt sich vom Eingriffsrecht des Staates in prekäre Familienverhältnisse zum Recht der sozialen Infrastruktur wie vorschulischen Bildung. Darüber verändert sich der Adressatenkreis der Jugendhilfe; nicht nur Randgruppen, sondern sämtliche Kleinkinder werden zu dessen potentiellem Adressaten.

63 OECD, Babies and Bosses (Fußn. 35), S. $103 \mathrm{ff}$.

$64 O E C D$, Babies and Bosses (Fußn. 35), S. 44 ff.

65 Dethloff, Gutachten 67. DJT (2008), A $131 \mathrm{ff}$.

66 Vgl. dazu Köbl, Welche Maßnahmen empfehlen sich, um die Vereinbarkeit von Berufstätigkeit und Familie zu verbessern?, JZ 1994, S. 840; auch in der Unfallversicherung: dies., in: Hofer/Klippel/Walter (Hrsg.), Perspektiven des Familienrechts (Fußn. 22), S. 997 ff.; Michaelis, Familienlastenausgleich in der Rentenversicherung, VSSR 1996, S. 135; ders., Familienleistungen in der Rentenversicherung, DRV 1997, S. 696.

67 Bradshaw/Mayhews, Family Benefit Packages, in: ders./Hatland (Fußn. 14), S. 97 ff. 
Das Jugendhilferecht, 68 aus der Armenpflege hervorgegangen, entwickelte sich im 20. Jahrhundert zu einem die Armenfürsorge mit polizeyrechtlichen Traditionen verbindenden Rechtsgebiet, das zunächst namentlich der Verwahrlosung von Kindern und Jugendlichen aus sozialen Randgruppen entgegenzuwirken hatte. Es trat in den Fällen auf den Plan, in denen die Familie nicht der „Hort der Menschenwürde“, sondern der Ort der Entrechtung und Erniedrigung von Kindern war. Dementsprechend sollte es das dem jeden Jugendlichen zukommende Recht auf Erziehung notfalls durch öffentliche Mittel, Funktionsträger und Institutionen zu verwirklichen helfen, soweit namentlich private Erziehung dem Jugendlichen vorenthalten wurde oder sich als unzureichend erwies. Jugendhilfe fand daher vor allem seinen Auftrag in der öffentlichen Kontrolle über die Familienverantwortung durch die Eltern. Erziehungsbeistandschaft oder Erziehung durch Pflegeeltern oder Heime waren die Möglichkeiten öffentlicher Einwirkung auf Elternverantwortung bis zum Entzug von Elternverantwortung und deren Übertragung auf öffentliche oder freie Träger.

Heute wird es vom Eingriffsrecht des Staates in prekäre Familienverhältnisse zum Recht der sozialen Infrastruktur und Teil des vorschulischen Bildungswesens. Der Adressatenkreis jugendhilferechtlicher Maßnahmen verändert sich dadurch. An die Stelle von Randgruppen tritt die Gesamtheit aller Kleinkinder. Potentiell wird so künftig jedem Kind jugendhilferechtlicher Schutz zuteil.

\section{Familienförderung und Chancengleichheit für Familien}

Diese Anstrengungen, die Elternschaft sozialrechtlich zu unterstützen wie zu begleiten, sind die Antwort der Gesellschaft auf die ,strukturelle Rücksichtslosigkeit von Gesellschaft und Staat gegenüber den Tatbestand der Elternschaft".69 Die Gleichheit von Familien und Nichtfamilien, die Chancengleichheit von Menschen aus unterschiedlichen sozialen Schichten sowie die Geschlechtergleichheit leiten bei Verwirklichung dieser Ziele. ${ }^{70}$ Sie ergeben sich aus der Einsicht, dass ohne diese umfang- und zahlreichen Hilfen eine Gesellschaft weniger Kinder zu erwarten hätte als mit ihnen. ${ }^{71}$

In der zeitgenössischen Familiensoziologie werden für eine auf Wahrnehmung der Elternverantwortung gerichtete Sozialpolitik vier Motive angegeben ${ }^{72}$ : nämlich die

68 Eichenhofer, Sozialrecht (Fußn. 30), Rdnr. 566 ff.; Münder, Frankfurter Kommentar zum SGB VIII: Kinder- und Jugendhilfe, 5. Aufl. Weinheim 2006, Einleitung Rdnr. 54.

69 Kaufmann, Zukunft der Familien im vereinigten Deutschland, Minden 1995, S. 11; Scheiwe, Kinderkosten und Sorgearbeit im Recht, Frankfurt 1999.

70 BMJFS, Familien zwischen Flexibilität und Verlassenheit, S. 429 ff.

71 Esping-Andersen, A Child-Centred Social Investment Strategy, in: ders., Why we need a New Welfare State?, Oxford 2002, S. 26 ff.

72 Bradshaw/Finch, Can Policy Influence fertility?, in: Han Emanuel (Hrsg.), Ageing and the Labour Market: Issues and Solutions; or are there?, Antwerpen/Oxford, 2006, S. 151 ff.; vgl. auch Gauthier, 
sozialrechtliche Gleichstellung von Familien- und Erwerbsarbeit, ${ }^{73}$ die positive Beeinflussung der Reproduktionsentscheidung der Eltern, die Förderung der Geschlechtergleichheit und die Verminderung der ökonomischen Lasten von Kind und Elternschaft. In der deutschen Diskussion käme noch die Sorge um die Zukunftsfähigkeit der auf den Generationenvertrag begründeten Sozialversicherung als weiteres Motiv hinzu. ${ }^{74}$

Anders als ein schlicht gefasstes Subsidiaritätspostulat glauben macht, besteht folglich keineswegs ein einfaches und gar hierarchisches Vorrang-Verhältnis zwischen der Eigen-, Familien- und Solidarverantwortung! Vielmehr schafft der Sozialstaat durch viele und unterschiedlichen Maximen folgende Regeln erst den Rahmen, innerhalb dessen sich Eigenvor- und Familienfürsorge entfalten sollen und können. Deren jeweiliger Rang wird daher von dem sozialstaatlichen Arrangement insgesamt bestimmt.

„Subsidiarität“ ist unter diesen Gegebenheiten nicht der Ausgangs-, sondern allenfalls ein - im Übrigen ziemlich vage umschriebener - Endpunkt, weil die letztlich bestimmende Zielvorstellung aller sozialstaatlicher Gestaltung. Dabei enthüllt der Rechtsvergleich trotz interessanter Unterschiede im Detail auffällige Parallelen im Grundsätzlichen wie Allgemeinen. Die Solidarverantwortung wird in der Weise wirkmächtig, dass der Eigenverantwortung Raum bleibt und zugleich die Familienverantwortung wahrgenommen werden kann. Dabei ist die Sozialpolitik aller Staaten mit der besonderen Schwierigkeit grassierender Kinderarmut und der Verelendung allein erziehender Frauen konfrontiert. ${ }^{75}$ Grundsicherung und Sozialhilfe expandieren ungewollt, ja entgegen der propagierten Familienpolitik. Hiergegen hilft vor allem die Erwerbsbeteiligung der Frauen bei gleichzeitiger öffentlicher Sicherung der Kinderbetreuung. ${ }^{76}$

Es erscheint allerdings müßig, der Frage nachzugehen, welche unter den vier Motiven das letztlich ausschlaggebende sei. Vielmehr liegt es weit näher, in deren Bünde-

The State and Family, A Comparative Analysis of Family Policies in Industrialized Countries, Oxford 1996.

73 Sichtbar vor allem bei der Anrechnung von Erziehung im Rentenrecht: Köbl, Familienleistungen in der Alterssicherung, DRV 2002, S. 686 ff.; vgl. auch Ebsen, Verfassungsanforderungen an den Familienlastenausgleich in den Alterssicherungssystemen - einige Überlegungen zur Rechtsprechung des BVerfG, DRV 2002, S. 697, 705 ff.

74 Borchert, Renten vor dem Absturz, Frankfurt 1993.

75 European Commission, Joint Report on Social Protection and Social Inclusion 2008, S. 6 ff.

76 European Commission, Joint Report on Social Protection and Social Inclusion 2008, S. 11; Whiteford/Adema, Combating Child Poverty, in OECD Countries: is work the answer?, European Journal of Social Security 8 (2006), S. 235; Hoelscher, What works? Preventing and reducing child poverty in Europe, European Journal of Social Security 8 (2006), S. 257; auf diesen Ansätzen beruht der UK New Deal for Lone Parents und die US-amerikanische Reform der Sicherung Alleinerziehender im Übergang von AFDC zu TANF (1994); dem hergebrachten Sozialleistungsprogramm AFDC (Aid to Families with Dependent Children) wurde entgegengehalten, auf der Idee der Familien- und Sozialleistungsabhängig zu beruhen (Pateman, in: Pierson/Castles (Hrsg), The welfare state (Fußn. 18), S. 137); das an die Stelle gesetzte Programm TANF (Temporary Assistance for Needy Families) verfolgt vier Ziele: Kinderbetreuung in eigenem Haus oder bei Verwandten zu fördern, Berufsbildung, Arbeit und Heirat anzuregen, nichtehelichen Geburten entgegenzuwirken und zur Bildung oder Aufrechterhaltung von Zwei-Eltern-Familien beizutragen. 
lung das eigentliche Movens der zeitgenössischen Reformen von Familienförderung zu erblicken. Denn die vielfache Legitimierung von Familienförderung ist der entscheidende Grund ihrer Verwirklichung im demokratischen Prozess.

\section{Fazit}

Unter den heutigen Bedingungen einer umfassenden medizinischen Beherrschbarkeit menschlicher Reproduktion sind Kind- und Elternschaft nicht mehr dem Walten der Natur, sondern der bewussten Entscheidung der Eltern zuzuschreiben. Angesichts dessen greifen die Gesellschaften bewusst gestaltend in diese Entscheidung ein. Dies geschieht aus sozialpolitischen Motiven, aber auch um der Lebensentwürfen junger Eltern entgegen- wie nachzukommen. Wenn Familie von der Naturnotwendigkeit zum Akt bewusster Entscheidung wird, deren Rahmenbedingungen sozialpolitisch gestaltbar sind und daher gestaltet werden, so enthüllt die zeitgenössische Familienpolitik ein neues Aufgabenfeld sozialer Förderung, das in diesen Jahren mit großem Nachdruck ausgebaut und erheblichen öffentlichen Mitteln weiter entfaltet wird. 77

Familienpolitik hat eine fundamentale, weil existentielle Dimension. In ihr geht es um Grundfragen der individuellen Existenz. Soll sich der Mensch als Teil einer Generationenfolge oder isoliertes, sein eigenes Leben führendes und sich darin erschöpfendes Wesen verstehen? Um keine geringere Problematik geht es bei Familiengründung und ausweitung. Bindungen zwischen Menschen, ob als Eltern oder im Verhältnis zu den Kindern, werden begründet, welche die Beteiligten ein Leben lang miteinander verbinden. In welchem Maße der einzelne Mensch sich auf eine derartige Lebensform einlässt und einstellt, ist anders als noch vor wenigen Jahrzehnten, zum Gegenstand einer rationalen und medizinisch beherrschbaren Lebensentscheidung geworden.

Aus Gründen der Freiheit und des Respekts vor der individuellen Entscheidung eines jeden Menschen hat die Gesellschaft jede denkbare Entscheidung hinzunehmen. ${ }^{78} \mathrm{Da}-$ her ist die Propagierung irgendeines Lebensentwurfes - anders als bei sozialstaatlichen Reformen auf anderen Sektoren und Gebieten ${ }^{79}$ - eines liberalen und demokratischen Staates von Anfang an und von Grund auf versagt. Die Entscheidung für und gegen die Familie und die Entscheidung für oder gegen eine Familie einer bestimmten Größe und Größenordnung ist daher eine höchstpersönliche Entscheidung. Vor diesem Hintergrund ist der demokratische Staat weder berufen noch befugt, dem Einzelnen bindende Vor-

77 Dies findet in der Formung innerfamiliärer Pflege (dazu Köbl, Sozialstaatsentlastung durch mehr Familiensolidarität, in diesem Band, S. 393 ff.) eine Entsprechung.

78 Vgl. auch Eichenhofer, Gutachten zum 64. DJT (2002), B 56 ff.; Fuchs, Empfiehlt es sich die rechtliche Ordnung finanzieller Solidarität zwischen Verwandten im Unterhalts-, Pflichtteils-, Sozialhilfe- und Sozialversicherungsrecht neu zu regeln?, JZ 2002, S. 785; BMJFS, 7. Familienbericht, Familie zwischen Flexibilität und Verlässlichkeit, August 2005, S. 427 ff.

79 Grundlegend de Swaan, In Care of the State, Cambridge 1988. 
schläge über die Gestaltung seines Lebens zu machen. Es wäre daher falsch, eine unter Billigung der Entscheidungsfreiheit für oder gegen die Familie als die gegenwärtig angestrebte Familienförderung mit einer die Bevölkerungszahl ausweitenden „Bevölkerungspolitik" in eins zu setzen.

Gerade wegen der nicht hintergehbaren Wählbarkeit der Lebensform Familie ist in einer demokratischen Gesellschaft nur eine solcher Familienpolitik konsensfähig, wenn ihre Ziele und Maximen tendenziell von allen Menschen gebilligt werden können, die zu den Fragen der familiären Existenz und des Ausmaßes der eigenen Familie zu unterschiedlichen Ergebnissen kommen können und dürfen. Familienpolitik wird daher vor allem dann erfolgreich betrieben werden können, wenn sie unterschiedliche Motive und Letztbegründungen für familiäres Leben zu bündeln vermag. Die konzeptionelle Offenheit von Familienpolitik ist also eine unverzichtbare Voraussetzung ihres Erfolges. Die Wahlfreiheit in der Wahrnehmung familiärer Aufgaben hat der Sozialstaat den Eltern ebenso zu sichern wie deren Entscheidungsfreiheit im Hinblick auf die Familiengründung. Von staatlicher Familienförderung darf kein Konformitätsdruck ausgehen - jedes denkbare Model familiären Lebens ist erlaubt und daher auch sozialrechtlich zu fördern. Die Vielfalt familiärer Lebensformen zu sichern, ist aber nur das eine; solche Vielfalt vor dem Hintergrund knapper öffentlicher Mittel zu realisieren, ist aber das andere höchst Schwierige. Die öffentliche monetäre Unterstützung familiärer Erziehung als sogenanntes Betreuungsgeld kann als sozialstaatliche Anerkennung der Gleichwertigkeit und -würdigkeit öffentlicher Kinderbetreuung gedacht und gerechtfertigt werden. Allerdings ist das Betreuungsgeld die kostengünstige Alternative zu öffentlicher Kinderbetreuung - je weiter jenes reicht, desto kostspieliger kann diese werden. Außerdem ist Betreuungsgeld eine Gegenleistung für Familienerziehung; soll deshalb dessen Zahlung davon abhängig gemacht werden, dass mit ihr anerkannte Erziehungsziele angestrebt oder gar erreicht werden?

Der Aufbau einer sozialen Familienförderung geschieht nicht nur, weil sich die Familien als benachteiligt sehen, obgleich sie umfassend Erziehungs- und Bildungsleistungen erbringen und damit für den Bestand des Sozialstaats eine schlechthin konstitutive Wirkung erfüllen. ${ }^{80}$ So ist die neue Familienpolitik nicht nur aus sich heraus begründet und zu rechtfertigen, sondern gewinnt ihren Sinn als Element zur Sicherung der institutionellen Voraussetzungen der sozialen Sicherung selbst. Familien, die ihrer Bestimmung folgen, erweisen sich damit für den Sozialstaat als ebenso elementar wichtig wie ein leistungsfähiger und starker Arbeitsmarkt, welcher der Entstehung von Sozialleistungsfällen begegnet, ihnen durch Integration in die Erwerbsgesellschaft entgegenwirkt und zugleich die Grundvoraussetzungen sozialen Transfers schafft. ${ }^{81}$ Familien

80 Rürup/Gruescu, Eine effektive und nachhaltige Familienpolitik führt zu wirtschaftlichem Wachstum, SF 2005, S. 273; Ruland, Familie und Alterssicherung, FamRZ 2004, S. 493; Seiler, Grundzüge eines öffentlichen Familienrechts, Tübingen 2008, S. $141 \mathrm{ff}$.

81 Sarfati/Bonoli, Labour Market and Social Protection Reforms in International Perspective, Altershot 2002. 
werden daher ähnlich wie Erwerbstätige durch den Sozialstaat gefördert; dessen Ausgabenprofil verändert sich jedoch, wendet sich von der letzten auf die erste Phase des Lebens. Das insgesamt künstliche Sozialrecht wirkt als zweite Schale des Rechts auf die vorfindliche gesellschaftliche Ordnung daher unmittelbar mobilisierend ein, auf dass Familie ihrer Bestimmung folgt und das Sozialleistungssystem selbst dauerhaft wirken kann! 


\title{
Die Familie im Gefüge der Solidargemeinschaften: Ein Ansatz soziologisch orientierter Rechtsvergleichung
}

\author{
Alexander Graser
}

Vorbemerkung

I. Die Rolle der Familie in sozialrechtsvergleichender Perspektive - ein Forschungsansatz

1. Sozialrecht, Gleichheit und Gemeinschaft

a) Sozialrecht als gleichheitsgenerierendes Recht 374

b) Sozialrecht als ,egalitärer Arm der Aufklärung“

c) Die (Bezugs-)Gemeinschaft als Prämisse der Gleichheitsgenerierung 376

2. Die Familie im Sozialrecht

a) Die Familie: Bezugsgemeinschaft oder Hindernis kollektiver Gleichheitsgenerierung?

b) Der Rückzug der Familie als Solidargemeinschaft im positiven Recht

c) Die bleibende Bedeutung der Familie als Solidargemeinschaft

3. Die Familie als Solidargemeinschaft in rechtsvergleichender Perspektive $\quad 379$

a) Die Familie im US-amerikanischen Recht 379

b) Gegenüberstellung von US-amerikanischem und deutschem Recht $\quad 380$

II. Erweiterungen des Forschungsansatzes 381

1. Das erweiterte Gefüge rechtlich verfestigter Solidargemeinschaften 381

a) Illustration des erweiterten Gefüges rechtlich verfestigter

Solidargemeinschaften

b) Die Bedeutung des erweiterten Gefüges für den zuvor vorgestellten Ansatz

2. Die Familie als ein (auch) gewillkürtes Verhältnis 384

3. Die Bedeutung sozialer Dienste 385

III. Dogmatik und Sozialrechtsvergleich $\quad 386$

1. Rechtswissenschaftliche Erkenntnisziele $\quad 386$

2. Rechtsvergleichende Erkenntnisziele 387

3. Zur (verborgenen) Bedeutung der Dogmatik in der (Sozial-)
Rechtsvergleichung

a) Dogmatische Ziele der Rechtsvergleichung 388

b) Die unverzichtbare (Hilfs-)Funktion der Dogmatik 389

c) Dogmatische Expertise als Proprium der Sozialrechtsvergleichung $\quad 390$ 